\title{
Galaxy Interactions and Strength of Nuclear Activity
}

\author{
S. M. Simkin \\ Michigan State University \\ East Lansing, MI 48824
}

\section{INTRODUCTION}

Almost a decade ago, the morphological similarities between patterns seen in Seyfert galaxies and those produced by gravitational forcing led to the suggestion that Seyfert activity might be fueled by material inflow induced by either a central bar or a perturbing companion (Simkin, Su, and Schwarz, 1980). More recent theoretical calculations suggest that such a feeding mechanism may involve a central bar-like, structure even if initially induced by the tidal effects of a companion galaxy (Noguchi, 1988a,b).

The recent literature on the prevalence of Seyfert galaxy companions, however, is somewhat confusing and, at initial glance, contradictory (Byrd, et al., 1987, Dahari, 1984, 1985, Fuentes-Williams, and Stocke, 1988, Keel, et al., 1985, Kennicutt, and Keel, 1984, Kollatschny and Fricke, 1989, Petrosian, 1982, and Petrosian and Turatto, 1986). Most studies find either a weak correlation between excess companions or none at all. All of these different studies are subject to different selection criteria, and most authors have attributed their disparate conclusion to selection effects (op. cit.).

An independent analysis of the material in these papers by the present author seems to suggest an excess of nearby companions for Seyfert 2 galaxies, but not for Seyfert 1 galaxies (with the term nearby subject to different defining criteria for different studies). On the other hand, two of the cited studies show no significant difference in excess companion density between the two Seyfert classes (Byrd, et al., 1987, and Kollatschny and Fricke, 1989). Both of these latter studies, however, deal with small numbers of objects.

\section{DIFFERENCES BETWEEN TYPE 1 and TYPE 2 SEYFERTS}

When all of the data in the papers cited above are combined, an interesting trend emerges. There are 34 pairs for the Seyferts with companions and $z \leq 4000 \mathrm{~km} \mathrm{~s}^{-1}$. This comprises roughly $40 \%$ of the known Seyferts with $z$ in this redshift range. Given the very different selection criteria of the different studies which have been included, it is difficult to evaluate just how representative these data are. However, twelve of these pairs contain Seyfert 1 galaxies and twenty two contain Seyfert 2's. Thus the ratio of Sey 1's to Sey 2's (approximately 1:2) is similar to that found for nearby, volume limited samples of Seyfert galaxies irrespective of their status as companions (Simkin, Su, and Schwarz, 1980). Although this does not prove the sample is unbiased, it does support its credibility.

Converting angular separation to projected physical separation using a Hubble distance based on $\mathrm{H}_{o}=100$ and ploting this against radial velocity difference for each pair gives the results shown in Figure 1. The plot demonstrates a fairly clear distinction between Seyfert 1 and Seyfert 2 galaxies. For the objects in this sample, $10(90 \%)$ of the Seyfert 1 galaxies are separated from their "companions" by projected distances of more than $75 \mathrm{kpc}$ while 18 (90\%) of the Seyfert 2's 
are separated by less than $75 \mathrm{kpc}$.

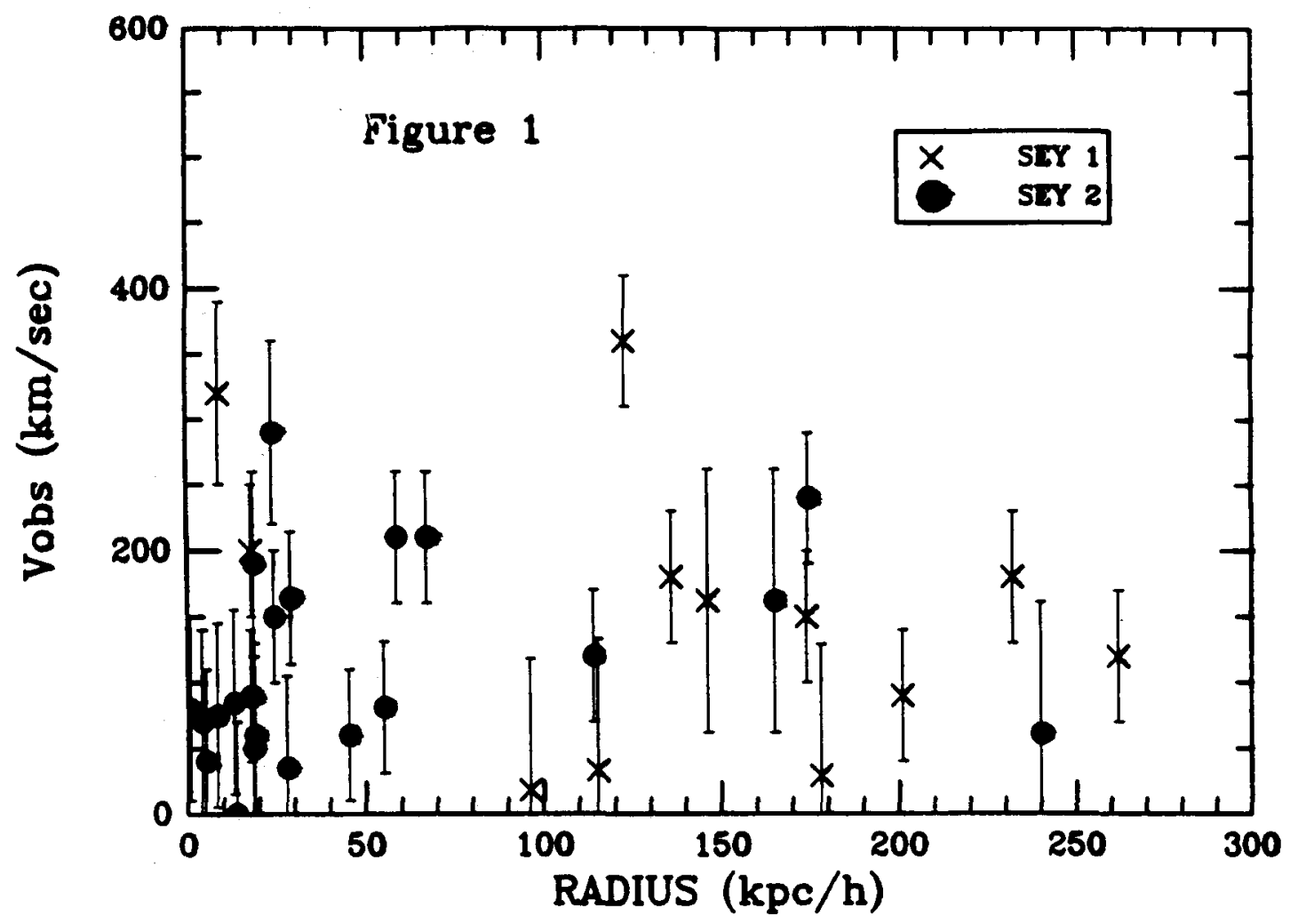

\section{DISCUSSION}

There are two straightforward interpretations of the difference between Seyfert 1 and Seyfert 2 galaxies shown in Figure 1: i) either most Seyfert 1 galaxies do not have companions (the plotted points represent chance projection) while Seyfert 2 galaxies do, or ii) there is a true physical distinction between the two types of galaxies and this distinction is related to the nature of the tidal pertubation they have experienced. The first possibility seems somewhat unlikely since, as noted above, the fraction of Sey1 and Sey2, galaxies with respect to those in a volume limited sample, is the same for those with "companions" as for those without. In addition, the selection criteria used in the studies from which these data were taken included a limit on velocity, thus sharply reducing the number of expected pairs attributable to projection effects.

The second possibility is supported by the fact that there is other, independent evidence for morphological differences between type 1 and type 2 Seyferts which may also be attributed to differences in the histories of their gravitational disturbances. Seyfert 1 galaxies have narrower spiral arm (or ring) widths than Seyfert 2 galaxies of similar physical size (Simkin et al., 1989, 1990). Again, there are two possiblities which immediately come to mind: i) the arm-ring features in the Seyfert 1 galaxies may be produced by gravitational disturbances leading to pattern speeds which generate more narrow features (sharper shocks) than those for the Seyfert 2's, or ii) the Seyfert 2 galaxies may have evolved from Seyfert 1's with their arm-ring features spreading out in width with age. 
This "aging" interpretation of arm width difference is unlikely, however, because the UVB colors for the disks of nearby Seyfert galaxies show no distinctions between type 1 and type 2, and the colors of stars produced in spiral arm shocks should redden with age (Simkin et al., 1990). Thus it is most likely that the much larger average projected separation found between Sey 1's and their companions than between Sey 2's and theirs, represents a real physical distinction.

One other interesting result can be obtained from the sample of Seyfert galaxies discussed here. If one assumes that all of the objects are true physical companions (in effect, using nuclear activity as a diagnostic of gravitational interaction), then a crude analysis gives the following mean physical parameters for the sample: mean mass $\sim 10^{12} \mathrm{M}_{o}$, mean size $\sim 30-40 \mathrm{kpc}$, mean $\mathrm{M} / \mathrm{L} \sim 8-10$ solar units. These are quite comparable to the values reported in this conference for the binary galaxy sample of Karechentsev, either an amusing coincidence or additional support for the interaction hypothesis.

\section{CONCLUSIONS}

Analysis of data in the literature for differential velocities and projected separations of nearby Seyfert galaxies with possible companions shows a clear difference in projected separations between type 1's and type 2's. This kinematic difference between the two activity classes reinforces other independent evidence that their different nuclear characteristics are related to a non-nuclear physical distinction between the two classes. The differential velocities and projected separations of the galaxy pairs in this sample yield mean galaxy masses, sizes, and mass to light ratios which are consistent with those found by the statistical methods of Karachentsev. Although the galaxy sample discussed here is too small and too poorly defined to provide robust support for these conclusions, the results strongly suggest that nuclear activity in Seyfert galaxies is associated with gravitational perturbations from companion galaxies, and that there are physical distinctions between the host companions of Seyfert 1 and Seyfert 2 nuclei which may depend both on the envronment and the structure of the host galaxy itself.

\section{REFERENCES}

Byrd, G., Sundelius, B., and Valtonen, 1987, Astron. and Ap., 171, 16.

Dahari, O., 1984, A.J., 89, 966.

Dahari, O., 1985, A.J., 90, 1772.

Fuentes-Williams, T. and Stocke, J. T., 1988, A.J., 96, 1235.

Keel, W.C., et al., 1985, A.J., 90, 708.

Kennicutt, R.C., and Keel, W.C., 1984, Ap. J. Lett., 279, L5.

Kollatschny W., and Fricke K.J., 1989, Astr. and Ap., 219, 34.

Noguchi, M., 1988a, M.N.R.A.S. 288, 635.

Noguchi, M., 1988b, IAU Collquium No. 96.

Petrosian, A.R., 1982, Astrofizika, 18, 548.

Petrosian, A.R., and Turatto, M., 1986, Astr. and Ap., 163, 26.

Simkin, S.M., Su, H-J., and Schwarz, M.P., 1980, Ap.J., 237, 404.

Simkin, S.M., Mehlberg, L., 1989, B.A.A.S., 21, 776.

Simkin, S.M., Geimer, S. and Mehlberg, L., 1990 in preparation 


\section{DISCUSSION}

Kochhar: In your picture what separates seyferts from nonSeyferts?

Simkin: All I was addressing was a mechanism for redistributing angular momentum to permit disk material to flow towards the nucleus. We need to study many systems (get rotation curves, simulate encounters with companions, etc.) to see if the difference between seyferts and other galaxies with significant redistribution of disk angular momentum is global or if both Seyferts and non-Seyferts have the same global properties but non-seyferts form "starbursts" while seyferts also have a "monster" at the center. 